\title{
Electrical and Optical Properties of Germanium-Doped Zinc Oxide Thin Films
}

\author{
Makoto Arita, Mayu Yamaguchi* and Masataka Masuda \\ Department of Materials Science and Engineering, Faculty of Engineering, Kyushu University, \\ Fukuoka 812-8581, Japan
}

Germanium-doped zinc oxide thin films with Ge content of $0 \sim 8.1$ at $\%$ were deposited by an RF magnetron sputtering. The electrical and optical properties of the films were investigated. The Ge doping caused the reduction of resistivity of the films, and at about $3 \%$ of the Ge content showed the minimum resistivity of about $2 \times 10^{-3} \Omega \mathrm{cm}$. With high content of Ge, however, the crystalline structure changed and the resistivity of the film increased.

(Received April 16, 2004; Accepted September 29, 2004)

Keywords: zinc oxide, germanium, thin film, electrical conductivity, visible light transparency

\section{Introduction}

In recent years, zinc oxide thin films have been attracting a considerable attention because of its potential applicability for new electronic devices ${ }^{1)}$ as well as for transparent electrodes used in flat panel displays or solar cell panels. ${ }^{2)}$ Zinc oxide has the advantage that it is an abundant and inexpensive compound material in comparison with indium tin oxide (ITO), which is used as transparent electrode at the present time.

Some undoped zinc oxide films show electrical conduction (resistivity of $4.6 \times 10^{-4} \sim 10^{-3} \Omega \mathrm{cm}$ ). ${ }^{2-5}$ ) The origin of the electrical conduction of undoped zinc oxide films is considered to be their nonstoichiometry such as interstitial zinc atoms and/or oxygen vacancies. So the conductivity of these films is easily changed by high temperature treatment or by aging in air and strongly depends on deposition processes. ${ }^{6,7)}$ In order to obtain higher and stable electrical conductivity derived from dopant ions, a considerable number of studies on impurity doped zinc oxide films have been done. ${ }^{8-16)}$

Among these works doping of group III elements were mainly reported and the lowest resistivity of $1.9 \times 10^{-4} \Omega \mathrm{cm}$ was obtained with an Al-doped zinc oxide film. ${ }^{9)}$ However, if the group III impurity doped-zinc oxide film is used in heterostructural devices including an a-Si layer, for example a-Si solar cell panel, an anxiety has been pointed out that the characteristics of the device are degraded by the diffusion of the impurity atom into the a-Si layer. ${ }^{17)}$

Doping of Ge, which is one of the group IV atoms, into zinc oxide is expected to be harmless to the use of the oxide in devices including a-Si layer. Furthermore, Ge ion may be more effective donor than the group III impurities if it could successfully substitute $\mathrm{Zn}$ ion sites and be activated in the oxide lattice. There is, however, only one report on the Gedoped zinc oxide films. Sato et al. investigated the Ge-doped zinc oxide thin films prepared by an RF sputtering system with an external solenoid coil and reported that the lowest resistivity of $7.4 \times 10^{-4} \Omega \mathrm{cm}$ was obtained using a sputtering target including 2 mass $\%$ of $\mathrm{Ge},{ }^{18)}$ but the true concentration of impurity in the deposited film was not shown.

*Graduate Student, Kyushu University
Impurity doped zinc oxide films show a maximum of conductivity at a proper dopant concentration, namely excess of impurity doping causes deterioration in the electrical property. With a view to improve the electrical property of impurity doped zinc oxide films, it is required to understand the relationship between various properties of the films and the true concentration of doped impurities.

In this work, Ge-doped zinc oxide thin films with $\mathrm{Ge}$ content of $0 \sim 8.1$ at\% were deposited by an RF magnetron sputtering method. Electrical, optical, and structural properties of the films were investigated. The concentration of $\mathrm{Ge}$ in the films was estimated by X-ray photoelectron spectroscopy (XPS) and the relationship between those properties and the Ge content was discussed. In order to deepen understanding on the conduction mechanism in the films, the temperature dependence of the electrical properties was studied as well.

\section{Experimental Procedure}

Germanium-doped zinc oxide films were deposited on fused silica glass with a conventional RF sputtering apparatus ULVAC BC2227. The deposited area was $12 \times 12 \mathrm{~mm}^{2}$. The base pressure of the chamber was $5 \times 10^{-4} \mathrm{~Pa}$, and the distance between the substrate and the target was $60 \mathrm{~mm}$. The target disks were prepared by sintering the mixture of $\mathrm{ZnO}$ (>99.95\%) and $\mathrm{GeO}_{2}(>99.99 \%)$ powders in air at $1273 \mathrm{~K}$. The content of $\mathrm{GeO}_{2}$ in the target disks ranged from 0 to $10 \mathrm{~mol} \%$. The sputtering gas was Ar and the flux was $3 \mathrm{sccm}$. The pressure during deposition was kept at $2.0 \mathrm{~Pa}$. The RF power of $30 \mathrm{~W}$ was applied.

To characterize structure of the deposited films XRD measurement was performed with $\theta-2 \theta$ scanning configuration using $\mathrm{Cu} \mathrm{K} \alpha$ radiation by RIGAKU Rota flex RU-200. The transmittance spectra of the oxide films were measured in the range from 200 to $1100 \mathrm{~nm}$ using Shimadzu UV160A UV-visible spectrophotometer. The transmittance was calibrated against a bare fused silica glass as a reference sample. Electrical properties of the films were measured under the temperature range from $10 \mathrm{~K}$ to R.T. The resistivity of the films was estimated by Van der Pauw method with four probes at constant current of $10^{-8} \sim 10^{-4} \mathrm{~A}$. Hall measurement was performed and the carrier concentration was estimated for the films having relatively high conductivity. 


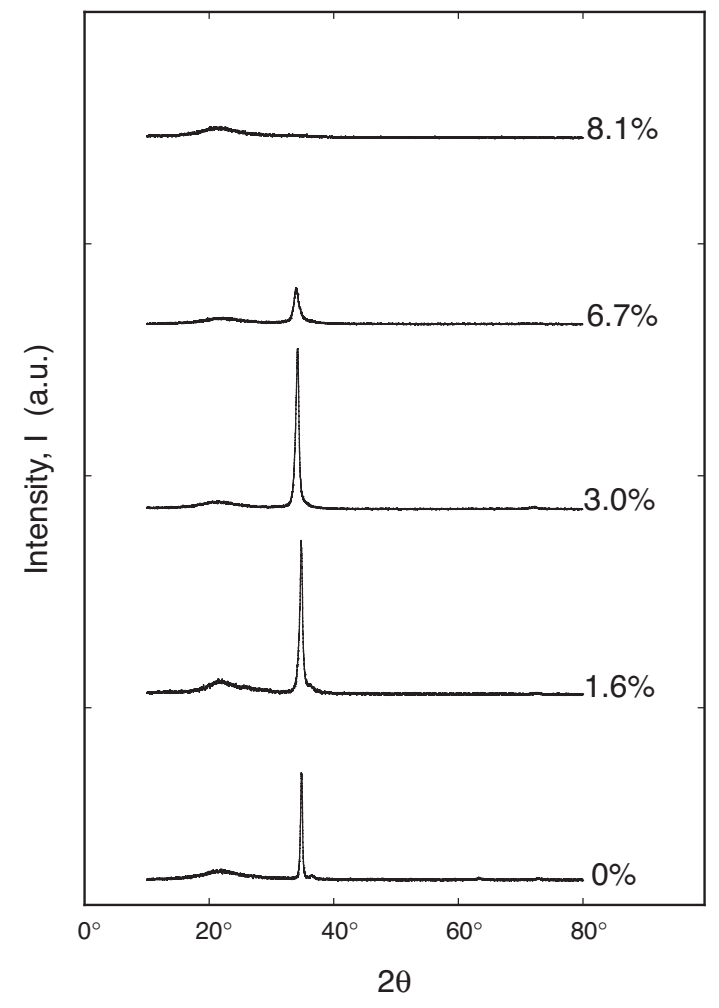

Fig. 1 XRD profiles for zinc oxide films with Ge content of 0, 1.6, 3.0, 6.7, and 8.1 at $\%$.

The true concentration of Ge in the oxide films was estimated by XPS measurement using PerkinElmer PHI 5600 ESCA system.

\section{Results and Discussion}

By the XPS analysis the concentration of $\mathrm{Ge}$ in the deposited oxide films was estimated at $0 \sim 8.1$ at $\%$. There was a tendency that the Ge content of the deposited films was lower than that of the sputtering target. The thickness of these films ranged from 190 to $450 \mathrm{~nm}$. Figure 1 shows the XRD profiles for the films with the Ge content of $0,1.6,3.0,6.7$, and $8.1 \%$. A broad peak around $21^{\circ}$ is due to diffraction from the silica substrate. For the films except for one with $8.1 \%$ of Ge content, a diffraction peak of wurtzite $\mathrm{ZnO}$ (002) was observed around $34^{\circ}$ in $2 \theta$ angle indicating a polycrystalline structure with c-axis orientation. The peak position of $\mathrm{ZnO}$ (002) slightly shifted toward a low diffraction angle as the Ge content became higher. The FWHM of the peak increased with an increase in the Ge content. On the contrary the Gerich film (Ge content of $8.1 \%$ ) showed no diffraction peak suggesting an amorphous structure. For all the samples, any diffraction peak, which would be derived from germanium oxide phases, was not observed. The atomic ratio of oxygen to metals in the film, $\mathrm{O} /(\mathrm{Ge}+\mathrm{Zn})$ calculated from XPS peak areas of $\mathrm{Zn} \mathrm{3d}$ and $\mathrm{O} 1 \mathrm{~s}$ is plotted in Fig. 2. The undoped film showed an oxygen-rich composition in comparison with stoichiometric $\mathrm{ZnO}$. The ratio of $\mathrm{O} /(\mathrm{Ge}+\mathrm{Zn})$ monotonically increased as the Ge content became higher.

Figure 3 shows the electrical resistivity, $\rho$ of the films measured at ambient temperature. The undoped zinc oxide

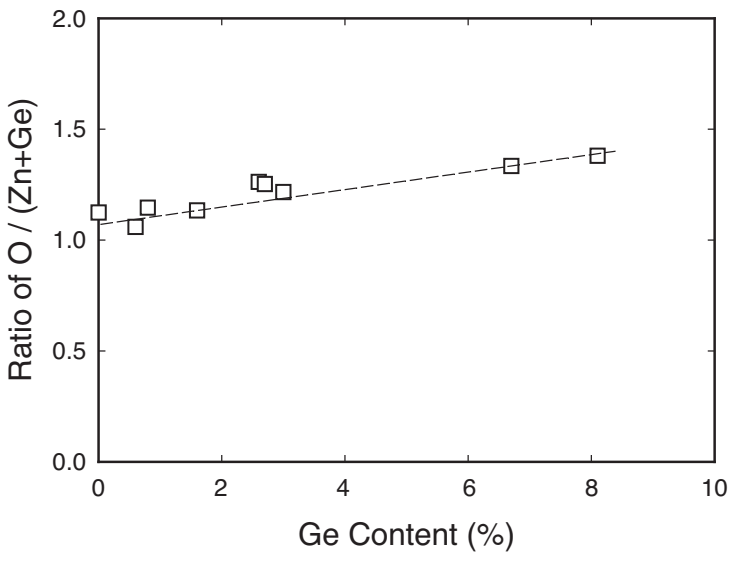

Fig. 2 Ratio of oxygen to metals $\mathrm{O} /(\mathrm{Ge}+\mathrm{Zn})$ in $\mathrm{Ge}$-doped zinc oxide films.

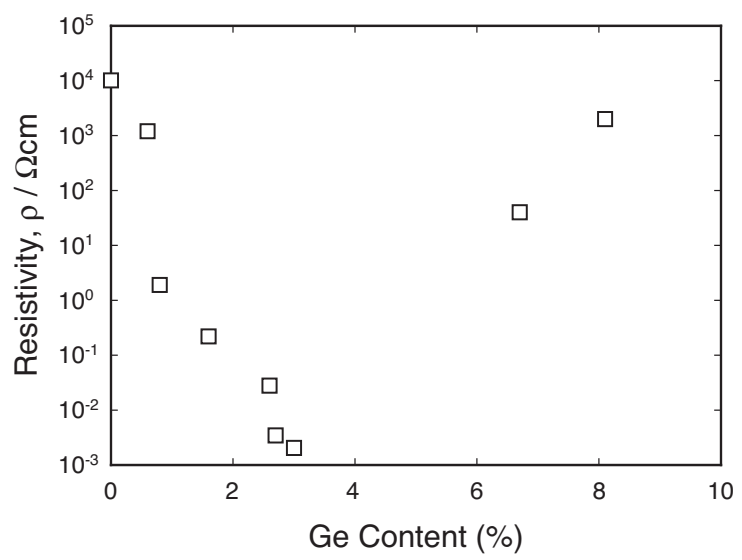

Fig. 3 Resistivity of Ge-doped zinc oxide films measured at ambient temperature.

film showed the relatively high resistivity of $1.0 \times 10^{4} \Omega \mathrm{cm}$. The resistivity of the film decreased as the Ge content increased up to $3 \%$, and we obtained the resistivity of about $2 \times 10^{-3} \Omega \mathrm{cm}$. On the other hand, with considerably high $\mathrm{Ge}$ content $(\geq 6.7 \%)$, the resistivity rose again and approached a value over $1.0 \times 10^{3} \Omega \mathrm{cm}$ at the Ge content of $8.1 \%$. Sato et al. investigated the Ge-doped $\mathrm{ZnO}$ thin films prepared by an RF sputtering system with an external solenoid coil and reported that the lowest resistivity of $7.4 \times 10^{-4} \Omega \mathrm{cm}$ was obtained at 2 mass $\%$ of Ge content in the sputtering target (about 1.6 at\% of Ge content). ${ }^{18)}$ In our experiment, the minimum of electrical resistivity might be obtained at more than 3 at $\%$ of Ge content. The difference of these electrical properties would be attributed to the film structure, which depends on the sputtering methods.

The temperature dependence of conductivity measured in the temperature range between $10 \mathrm{~K}$ and $300 \mathrm{~K}$ is shown in Fig. 4. For the low conductive films the conductivity changed with temperature, which would be considered as a superposition of Arrhenius-type temperature dependence. On the other hand the high conductive films showed no remarkable dependence of the conductivity on temperature. Muller studied the effects of heat treatment on the electrical properties of indium oxide films. ${ }^{19)}$ It was reported that the 


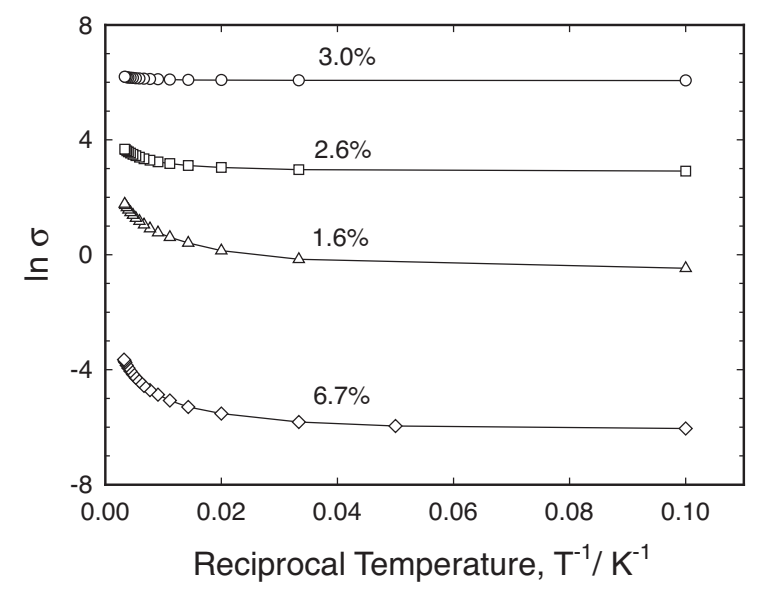

Fig. 4 Temperature dependence of electrical conductivity of Ge-doped zinc oxide films.

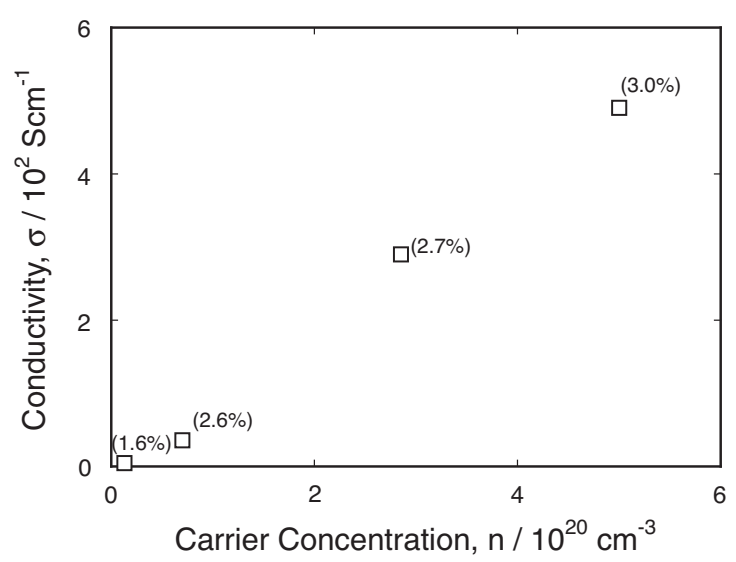

Fig. 5 Relationship between conductivity and carrier concentration.

temperature dependence of the carrier concentration and the Hall mobility disappeared with an increase in carrier concentration. Thus, for the highly conductive films, the conduction of electron may occur through degenerated band structure. On that occasion, it would be also implied that the donor states below the bottom of the conduction band broaden.

Figure 5 shows the relationship between the conductivity and the carrier concentration obtained by Hall measurement. For the poor conductive films exact value could not be evaluated by the apparatus. The negative Hall coefficient was observed indicating n-type conduction. For the films having relatively high conductivity, the Hall mobility showed no significant variation with a change of the Ge content and the conductivity was proportional to the carrier concentration. Thus, the improvement of conductivity would be governed mainly by the increase in the carrier concentration by the Ge doping.

The transmittance spectra for the films are shown in Fig. 6. The spectrum for the undoped zinc oxide film displayed the fundamental absorption edge at about $400 \mathrm{~nm}$ and the transmittance for the visible light was about $80 \%$. The optical band gap energy, $E_{\mathrm{g}}$ for direct optical transition of $4.9 \times 10^{-19} \mathrm{~J}(3.1 \mathrm{eV})$ was estimated by the eq. (1).

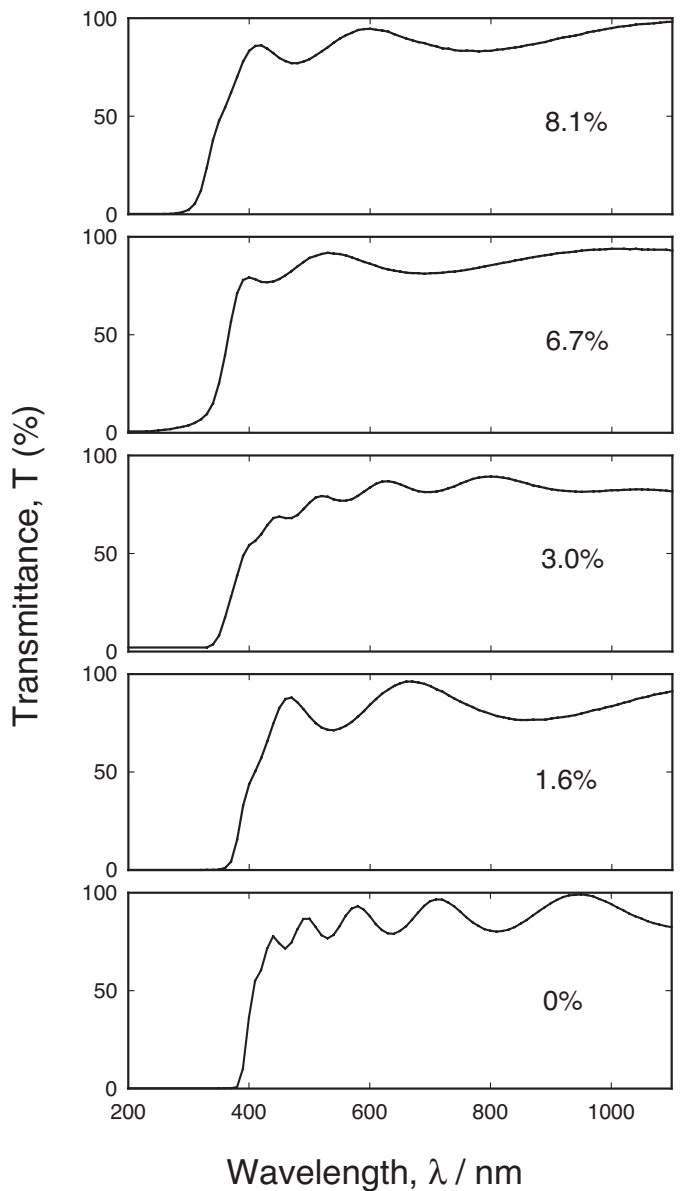

Fig. 6 Transmittance spectra for zinc oxide films with Ge content of $0,1.6$, $3.0,6.7$, and 8.1 at $\%$. The transmittance is normalized to the value for the film thickness of $400 \mathrm{~nm}$.

$$
\alpha \propto\left(h v-E_{\mathrm{g}}\right)^{0.5} / h v
$$

Here, $\alpha$ is the absorption coefficient calculated from the transmittance, and $h v$ the photon energy. The transmittance spectra of the Ge-doped oxide films are also showed in Fig. 6. These spectra showed oscillations in the high transmittance region, which were attributed to the interference of light in the oxide layer. The fundamental absorption edge shifts to shorter wavelength as the Ge content increases. The films that have Ge content of around 3.0\% showed slightly low transmittance particularly near the absorption edge. On the other hand the films with relatively lower and higher Ge content showed about $80 \%$ of transmittance in the visible region comparable to that of the undoped zinc oxide film. The degree of the declination in transmittance near the absorption edge corresponds to the electrical conductivity of the film.

Figure 7 summarizes the optical band gap energies for the films estimated by eq. (1). The $E_{\mathrm{g}}$ value increases from $4.9 \times 10^{-19} \mathrm{~J}$ for the undoped film to $5.8 \times 10^{-19} \mathrm{~J}(3.6 \mathrm{eV})$ at the Ge content of $8.1 \%$, as the Ge content increases. Lu et $a l$. investigated titanium-doped zinc oxide films deposited by a reactive magnetron co-sputtering method. ${ }^{20)}$ It was reported that the XRD diffraction peak from the $\mathrm{ZnO}$ (002) plane shifted to a lower angle and the $E_{\mathrm{g}}$ value increased with increasing the doping amount of $\mathrm{Ti}$ in the zinc oxide films. So the increase in the $E_{\mathrm{g}}$ value of germanium-doped zinc oxide 


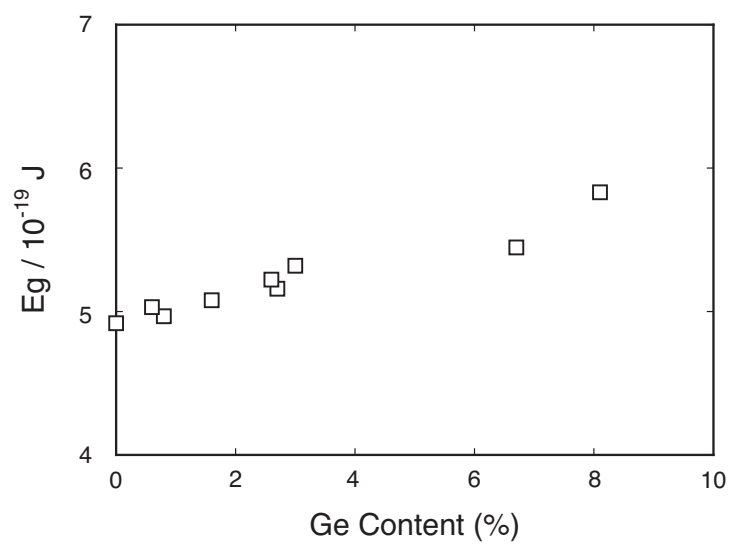

Fig. 7 Optical band gap energy for Ge-doped zinc oxide films.

films would be attributable to the lattice constant change of $\mathrm{ZnO}$ structure.

Since the ratio of $\mathrm{O} /(\mathrm{Ge}+\mathrm{Zn})$ rose monotonically with the increase in the Ge content (Fig. 2), it would be considered that most of the doped $\mathrm{Ge}$ atoms do not exist as divalent ions substitute the $\mathrm{Zn}^{2+}$ ion sites in $\mathrm{ZnO}$ lattice, but as tetravalent or trivalent ion species with higher oxygen ratio such as $\mathrm{GeO}_{2}$ in the film. Then, only the Ge atoms successfully substitute the $\mathrm{Zn}$ ion sites in $\mathrm{ZnO}$ lattice would act as donors. The number of them may increase with the increase in the doped Ge content. However, in the case of the heavily doped films, the crystalline structure begins to change and causes the decrease in electrical conductivity. In the case of zinc oxide films with excess aluminum doping, nonconductive $\mathrm{Al}_{2} \mathrm{O}_{3}$ clusters form in the films. ${ }^{21,22)}$ The $\mathrm{Al}_{2} \mathrm{O}_{3}$ clusters in the $\mathrm{ZnO}$ crystalline structure cause lattice disorders, which act as carrier traps and increase the electrical resistivity. So it is supposed that the films with considerable amount of $\mathrm{Ge}$ content include insulate $\mathrm{GeO}_{2}$ clusters and the carrier concentration of the films decreases consequently. This explanation is supported by the result that the film with the Ge content of $6.7 \%$ showed the temperature dependence again (Fig. 4). With the excess doping of Ge, the mobility of the films is considered to be reduced by the change of crystalline structure. Thus the decrease of electrical conductivity of the heavily doped films could be attributed not only to the reduction of the carrier concentration but also to the decrease in mobility.

\section{Conclusion}

Germanium-doped zinc oxide thin films with Ge content of
$0 \sim 8.1$ at\% were deposited by an RF magnetron sputtering. The doping of Ge caused reduction of resistivity of the films. At about $3 \%$ of Ge content the minimum resistivity of about $2 \times 10^{-3} \Omega \mathrm{cm}$ was obtained. The improvement of conductivity would be attributed to an increase in the carrier concentration by the Ge doping. With high content of Ge, however, the crystalline structure changed and the resistivity of the film increased. The optical transmittance of the high conductive films slightly reduced near the absorption edge in the visible region. The optical band gap energy was systematically increased with the increase in the Ge content.

\section{REFERENCES}

1) T. Yamamoto and H. Katayama-Yoshida: Jpn. J. Appl. Phys. Part2: Lett. 38 (1999) L166-169.

2) J. Aranovich, A. Ortiz and R. H. Bube: J. Vac. Sci. Technol. 16 (1979) 994-1003.

3) J. B. Webb, D. F. Williams and M. Buchanan: Appl. Phys. Lett. 39 (1981) 640-642.

4) T. Minami, H. Nanto and S. Takata: Appl. Phys. Lett. 41 (1982) 958960.

5) R. E. I. Schropp and A. Madan: J. Appl. Phys. 66 (1989) 2027-2031.

6) A. P. Roth and D. F. Williams: J. Appl. Phys. 52 (1981) 6685-6692.

7) T. Minami, H. Nanto, S. Shoji and S. Tanaka: Thin Solid Films 111 (1984) 167-174.

8) T. Minami, H. Sato, H. Nanto and S. Takata: Jpn. J. Appl. Phys. 24 (1985) L781-784.

9) T. Minami, H. Nanto and S. Takata: Jpn. J. Appl. Phys. 23 (1984) L280-282.

10) M. Arita, H. Konishi, K. Matsuda, M. Masuda and Y. Hayashi: Mater. Trans. 43 (2002) 1142-1145.

11) T. Minami: MRS Bulletin 25 (2000) 38-44.

12) K. Ellmer and R. Wendt: Surf. and Coationgs Technol. 93 (1997) 21-26.

13) A. F. Aktaruzzaman, G. L. Sharma and L. K. Malhotra: Thin Solid Films 198 (1991) 67-74.

14) K. Ellmer, F. Kudella, R. Schieck and S. Fiechter: Thin Solid Films 247 (1994) $15-23$.

15) J. Hu and R. G. Gordon: J. Appl. Phys. 71 (1992) 880-890.

16) K. H. Kim, K. Park and D. Y. Ma: J. Appl. Phys. 81 (1997) 7764-7772.

17) T. Minami, H. Sato, H. Nanto and S. Takata: Jpn. J. Appl. Phys. 25 (1986) L776-779.

18) H. Sato, T. Minami and S. Takata: J. Vac. Sci. Technol. A 11 (1993) 2975-2979.

19) H. K. Muller: Phys. Status Solidi 27 (1968) 723-731.

20) Y. M. Lu, C. M. Chang, S. I. Tsai and T. S. Wey: Thin Solid Films 447448 (2004) 56-60.

21) H. Kim, A. Pique, J. S. Horwitz, H. Murata, Z. H. Kafafi, C. M. Gilmore and D. B. Chrisey: Thin Solid Films 377-378 (2000) 798-802.

22) J. Hu and R. G. Gordon: J. Appl. Phys. 71 (1992) 880-890. 